\title{
BHसU
}

\section{Long-term practice of animal vaccination against dermatophytosis}

\author{
M. G. Manoyan, V.V.Sokolov, T.Y. Bukanova and A. N. Panin
}

Federal State Institution "The All-Russian State Center for Quality of Veterinary Drugs and Feeds" (FSI "VGNKI”) Moscow, Russia

Current approaches to control the animal dermatophytosis are based mainly on the using of chemotherapeutic drugs while the immunogenic medicines (vaccines, immunomodulators) have dropped out of sight.

Here we describe the Russian experience and prospects for the use of immunogenic medicines against dermatophytosis. The era of immunization against dermatophytosis was discovered by successful vaccine LTF-130 against cattle trichophytosis elaborated in USSR in 1960 yy by A.Kh. Sarkisov and co-workers.

Currently the vaccines in Russia are the primary means for both treatment and prevention of dermatophytosis while the chemotherapeutic drugs are used mainly as the auxiliary external means.

Nowadays a variety of the vaccines intended for various animal species are elaborated and introduced to the clinical practice in Russia: LTF-130 against trichophytosis in cattle, Microderm against dermatophytosis in small animals (cats, dogs, rodents, fur animals ) and horses. Those vaccines contain alive highly-immunogenic fungal strains which are attenuated and safe. The fullvalue fungal cells provoke intense immune response which is both protective and healing.

Also developed and introduced into veterinary practice inactivated vaccines against dermatophytosis animals, such as Vakderm and Polivak. The strategic significance of the vaccines is that they allow to conduct mass preventive immunization which reduces the incidence of dermatophytosis both in animals and mediately in humans.

The responsible animal-owners prefer to prevent the infection rather than pay money for costly treatment.

The proper administration of the vaccines is the key factor in defending humans against zoonotic dermatophytosis. Moreover the vaccines are wellproved means for the therapy providing the effective treatment without timetaking and expensive procedures.

The clinical efficiency of the mentioned vaccines is well-established and approved by the practical usage for over 40 years. All the officially allowed vaccines are monitored by governmental regulators for compliance with the quality and safety standards. A number of researches focused on vaccination and immunogenesis were published in domestic Russian issues but their international impact seems to be negligible.

We resume the vaccines possesses the great potential and abilities for prevention and treatment of animal dermatophytosis and reducing the disease incidence in humans.
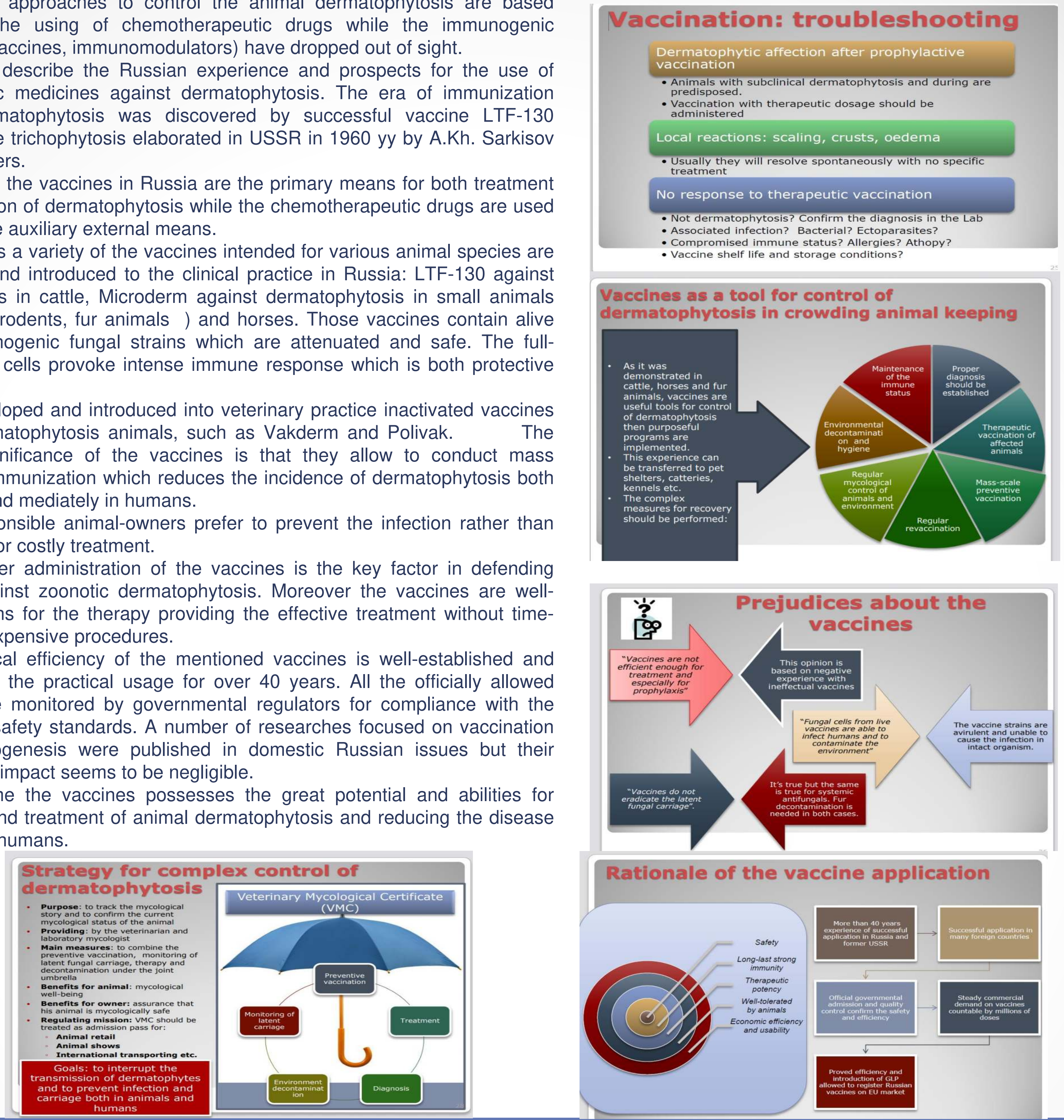

\section{CONCLUSION}

The vaccines possesses the great potential and abilities for both prevention and treatment of dermatophytosis in pets and farm animals.

- The vaccines demonstrate a number of advantages in comparison with systemic antifungals such as safety, usability and economical benefits.

- Immunoprophylaxis represents an extremely important tool for control of both animal and mediately human dermatophytosis.

- The commercial availability of the vaccines as well as awareness in the field of vaccination should be improved

We believe that international collaboration of veterinary mycologists can improve dramatically our experience in the field of combating fungal diseases.

\section{References on Russian vaccines}

- Manoyan MG, Panin AN, Letyagin KP. Effectiveness of microderm vaccine against dermatophytes in animals. Vet Dermatol 2000; (11 Suppl 1): 59.

- Manoyan M.G., Ovchinnikov R.S., Panin A.N. Therapeutic and prophylactic efficiency of a new vaccine "Equivac" against dermatophytosis in horses. Veterinary dermatology. - 2008. - vol. 19. - suppl. 1. - p. 77

- Manoyan M.G., Ovchinnikov R.S., Gaynullina A.G., Panin A.N. Large-scale vaccination and elimination of latent fungal carriage as a main measures for control of zoonotic dermatophytoses. 31st World Veterinary Congress - Prague, Czech Republic, 17-20 September 2013. Proceedings and Abstracts CD. - Manoyan M.G., Ovchinnikov R.S., Gaynullina A.G., Panin A.N. Commercial vaccines as a main tool for treatment and prevention of animal dermatophytosis in Russia. 31st World Veterinary Congress - Prague, Czech Republic, and etc. 